\title{
Practicalities of Targeted Radiotherapy in Non-Radiotherapy Departments for Breast Tumor Treatment
}

\author{
Khan $S^{1}$ and Dommett $\mathrm{D}^{2 *}$ \\ ${ }^{1}$ Physics, Surrey University, UK \\ ${ }^{2}$ Physics, Southend NHS Trust, UK
}

*Corresponding author: David Dommett MSc, Physics, Southend NHS Trust, Southend, UK, Tel: 07749 701889; Email: David.Dommett@southend.nhs.uk

\section{Case Report \\ Volume 4 Issue 3}

Received Date: July 13, 2020

Published Date: July 27, 2020

DOI: $10.23880 /$ crij-16000174

\section{Abstract}

Background: The National Institute for Health and Care Excellence (NICE) has several times reviewed the use of IORT with a view to approves the use of Targit intrabeam radiotherapy as a treatment option for people with early breast cancer. There have been some reservations from the Royal Collage of Radiologists (RCR) and concern from certain parties, even though NICE has agreed that intra-operative radiotherapy (IORT) would improve patients' quality of life. IORT offers accelerated partial breast irradiation (APBI) and intraoperative boosting for patients affected by breast cancer. Importantly it may reduce the risk of major coronary events (MCE) from reduced radiotherapy treatment as Darby SC, et al. has shown that there is a 7.4\% increase in MCE per Gray (Gy). There are numerous 'hidden' advantages such as travel time to and from the treatment centre, reduced transport costs as well as time saving to the individual and their carer's. These advantages along with the reduced shielding requirements compared to conventional radiotherapy and the growth in the private sector, mean we can see that the implementation of IORT could become a growth industry. Certainly there has been increasing interest and demand from patients for this kind of treatment. In this paper we aim to discuss and provide solutions to setting up a Targit IORT (Zeiss) system in a non-radiotherapy department.

IORT is routinely used to treat breast cancer in the USA (North and South America), the Middle East, Germany and other European countries and was recently approved for use in Australia in 2015.

System: The Zeiss manufactured system consists of a miniature, high dose rate, and low energy 50kV x-ray source (XRS). $0.05 \mathrm{~mA}$ electrons are accelerated and delivered with a $10 \mathrm{~cm}$ long $3.2 \mathrm{~mm}$ diameter probe with an Au target inside various size spherical applicators to match the tumour size. The applicator is placed in the tumour bed and the effective energy delivered is approximately $20 \mathrm{keV}$ or less with the dose falling off at $(1 / \mathrm{r} 3)$.

Keywords: Targit intrabeam radiotherapy; Breast cancer; Electron beam; Ionising radiation; Node biopsy; Isotropy

Abbreviations: NICE: National Institute for Health and Care Excellence; RCR: Royal Collage of Radiologists; IORT: Intra-Operative Radiotherapy; XRS: x-ray Source; APBI: Accelerated Partial Breast Irradiation; MCE: Major Coronary Events; LINAC: Linear Accelerator; OSL: Oncology Systems Ltd; EPD: Electronic Personal Dosimeter; IR: Ionising Radiations; RBE: Relative Biological Effectiveness; MPE: Medical Physics Expert.

\section{Introduction}

Developed in 1998 Targeted intraoperative radiotherapy is a technique for treatment of the tumour bed after wide local excision (lumpectomy) of breast cancer. Depending on the stage and grade of the tumour, radiotherapy can be recommended following surgery, as radiotherapy is used to kill off any microscopic spread of cancerous tissue beyond 
the tumour. With external beam radiotherapy, the whole breast is treated with 40.05Gy delivered in 15 fractions via a Linear Accelerator (LINAC) over 3 weeks - ensuring the tumour bed is irradiated $[1,2]$.

IORT aims to deliver a high dose of radiation (20Gy) in an isotropic manner precisely to the targeted area with minimal exposure of surrounding tissues, by delivering radiation from the site of the tumour immediately post-surgery. A probe used to generate $\mathrm{x}$-rays is placed within an applicator that approximates to the tumour size and the treatment is delivered for between 20 and 45 minutes. Following this, the applicator is removed, the wound closed and the patient returns to recovery and can often return home that evening [3].

The IORT system beams electrons down a probe to a gold tip. By slightly processing the electron beam around a gold target in the tip (rather like a spinning top or gyroscope), $\mathrm{x}$-rays are generated in all directions. Those x-rays passing back down the probe are monitored by an ion chamber near the electron source, allowing both output and beam direction to be monitored [4].

The aim of the study is to show that a mobile radiotherapy system for breast tumour treatment can be easily implemented in a small non radiotherapy hospital, the radiation safety are not a hindrance or too costly to prevent the service and benefit these patients.

\section{The IORT Probe and Treatment Process}

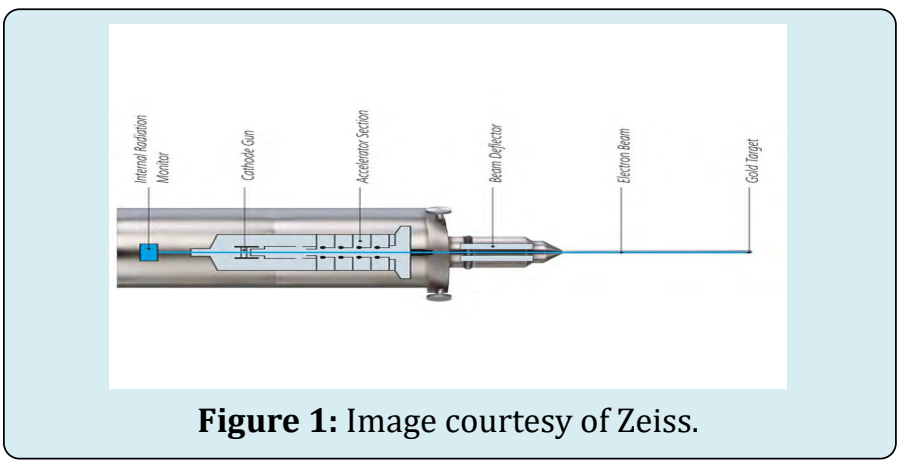

The Following Images Demonstrate the IORT Treatment Process

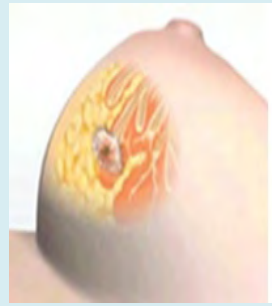

Figure 2: The position of the tumour is determined.

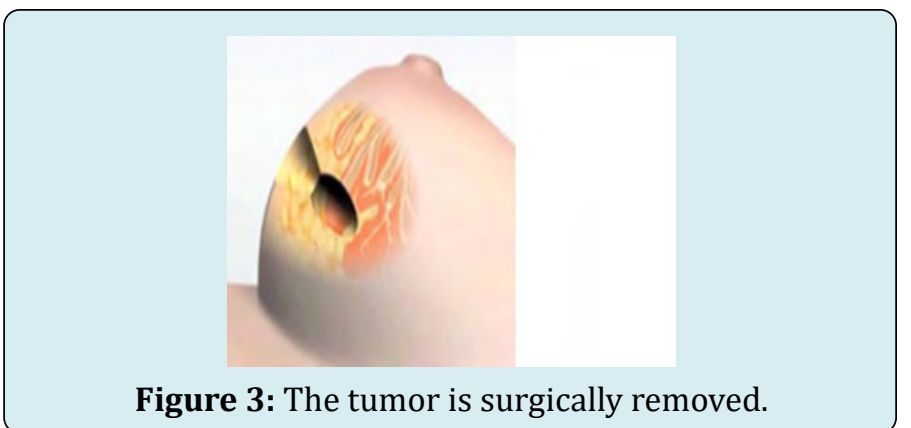

Figure 3: The tumor is surgically removed.
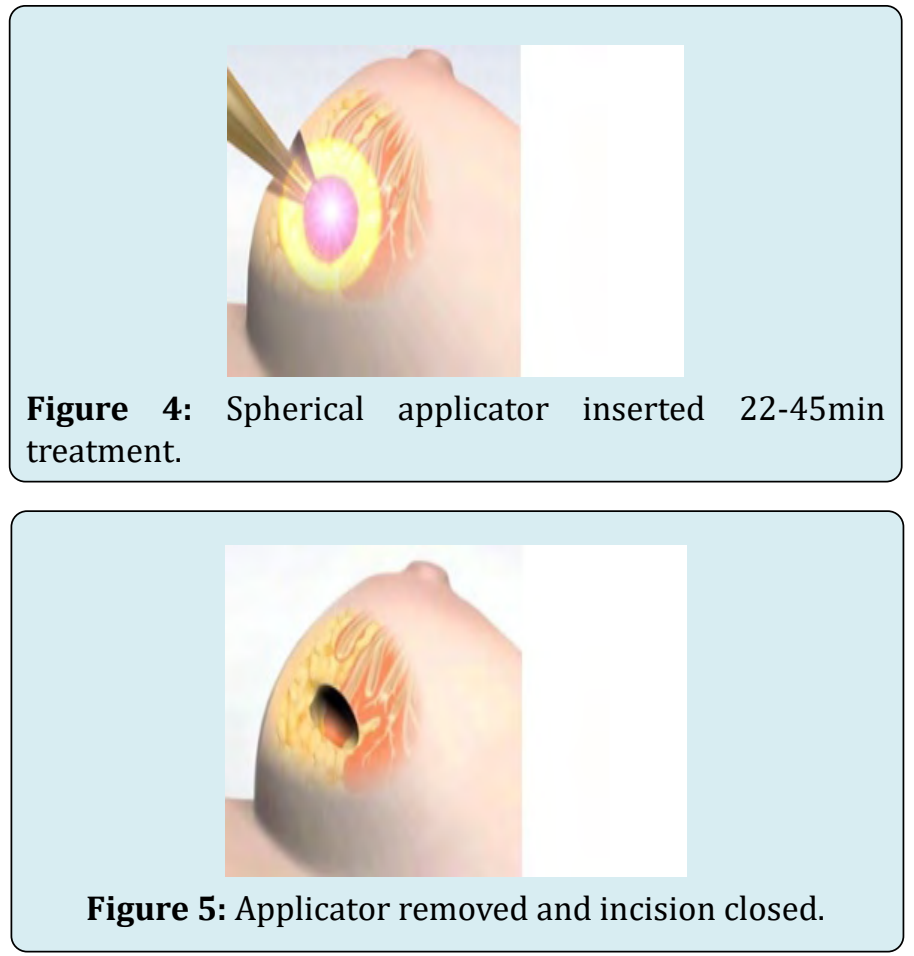

Due to the higher ionization density caused by soft X-ray radiation in the tissue, the relative biological effectiveness (RBE) of low-energy X-rays on tumor cells is higher when compared to high-energy X-rays or gamma rays which are delivered by linear accelerators. As the radiation which is produced by mobile radiation systems has a limited range the shielding requirements are lower than for a Linear Accelerator which makes it suitable for some theatres / departments.

The 5-year TARGIT-A trial, which recruited 3451 patients showed that giving TARGIT at the time of lumpectomy in ER+ $\mathrm{PR}+$ patients, aged 45 years and over, gives results similar to whole breast radiotherapy in terms of local cancer control, with fewer non-breast cancer deaths and a trend for lower overall mortality. The high installation and running costs of linear accelerator facilities should be compared with those of IORT, as well as the other benefits to patients. Many cancer centres cover a large geographical area and the time and 


\section{Clinical Radiology \& Imaging Journal}

distance commitment for some patients can be extreme. This is why many hospitals believe that IORT offers a viable alternative.

\section{Materials and Methods}

The first consideration is purchasing or hiring a system. Initial costs are about $£ 500 \mathrm{k}$ for the system, with additional costs for shielding and some ancillary equipment. Zeiss and Oncology Systems Ltd (OSL) provide a comprehensive package for 6 monthly source calibrations along with full servicing. Hiring a unit is cheaper in the short term, and would be given the same full support. In our view the purchased systems have been fairly robust, but it should be noted there is no insurance available for a damaged probe - replacement at a cost of approximately $£ 100 \mathrm{k}$ is the only option [5].

The next consideration is the theatre to be used. Despite the x-ray energy, dose rates in the room means it has to be designated as a controlled area and shielding $(2.5 \mathrm{~mm}$ lead equivalent) must be provided for staff remaining in the room. Normally this is positioned before the main doors, but equipment set-up may not allow for this and additional shielding may be required. As with any treatment involving ionising radiation room surveys are required and in many hospitals have to include rooms above and below as well as surrounding. It should be noted the short treatment times and general construction of buildings means there is rarely an issue outside the theatre. Flexible lead-equivalent shielding can be placed over the treatment site to reduce dose rates, and this is recommended. Doses should be measured at various points in the room and the surrounds and measures put in place to reduce dose if required. This is generally done using survey meters with the results recorded in $\mu \mathrm{Sv} / \mathrm{hr}$, but can also be done with ion chambers. Calculation of area designation can be done using these results and estimates of number of treatments to be performed.

IORT involves a normal wide local excision with possible sentinel node biopsy (often done using technicium- $99 \mathrm{~m}$ given a few hours pre-surgery), followed by the IORT treatment. Choosing the correct applicator, attaching it to the probe and placing in the patient adds an additional 5-10 minutes to theatre time, followed by between 25 to 50 minutes for treatment depending on applicator size. This extra time has to be considered within the department's workload, and also the cost of staff who cannot be carrying out theatre work during this time. Additional measures need to be taken with any radioactive node tissue removed. Applicators (made from stainless steel and ULTEM (Polyetherimide) designed to attenuate the radiation vary in size from $15 \mathrm{~mm}$ up to $50 \mathrm{~mm}$ in $5 \mathrm{~mm}$ sizes. These require sterilisation between each use, and so for common sizes it is recommended to have more than one applicator.
Access control is important - especially as many staff may no longer take note of warning signs and so lockable doors are useful, along with suitable staff training. Staff monitoring for the radiographer, anaesthetist and physicist who remain in the theatre during treatment is preferably done by an Electronic Personal Dosimeter (EPD) which provides an instantaneous readout - and can allow for staff changes such as the anaesthetist as long as clear records are kept. As long as the recommended shielding is used, results are generally low, and often zero, over the course of the treatment. Certain key staff may be issued (or already have) film badges to provide additional monitoring. Staff results from several centres have shown no significant increase in staff dose.

As the theatre is designated a controlled area under the Ionising Radiations Regulations 2017 (IRR17) [6], local rules are required, which can be combined with recommendations for sentinel node radioactive handling if staff have concerns. It is important to develop a suitable working protocol covering set up, calibration, storage, data storage, consent, sign-offs and treatment. In addition to the critical exam, commissioning is required and an independent audit of the results. Commissioning involves checking the depth dose measurements and isotropy of the applicators, along with other checks. All relevant legislation has to be adhered to, including IRR17 and the Ionising Radiations (Medical Exposure) Regulations (IR(ME)R2017) [7], which means operator lists have to be generated, risk assessments completed and so on. Both pieces of legislation require suitable training, so all staff should understand basic radiation safety, and have read and understood the local rules. However, during the procedure generally only the anaesthetist, a radiographer and sometimes a physicist are present and these must be clear on the safety requirements. Those operating the equipment must have suitable training in its use. Staff training of surgeons, nurses, physics and theatre staff is very important as many have little experience of the precautions required when using ionising radiation.

\section{Results of a Room Survey}

The image below is the result of a several room surveys that gradually improved on the final layout. These surveys were carried out using a Mini Smartlon. Under the worst case scenario basis, the first survey was just the bare probe, however the results within the room were higher than anticipated, and so a more realistic scenario was created. A phantom patient was designed, and after this, a further survey was done with the recommended lead equivalent shielding placed over the 'treatment site' (Figure 6 \& Table 1). 


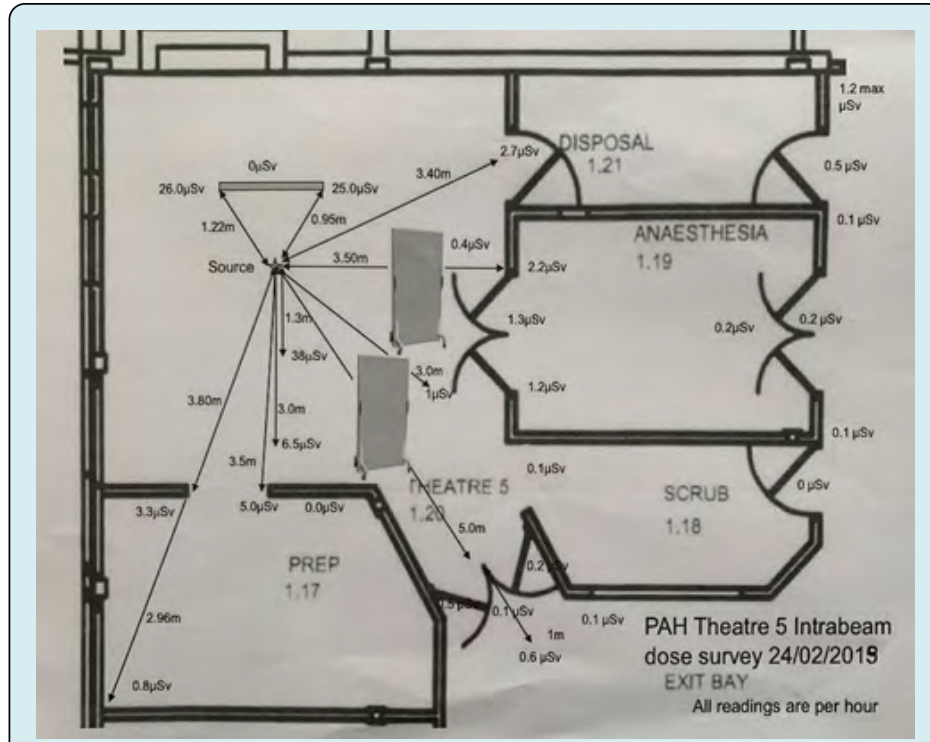

Figure 6: PAH Theatre 5 Intrabeam dose survey.

The following table shows results at some example points as a comparison of differing set-ups.

\begin{tabular}{|c|c|c|c|}
\hline Position $\downarrow /$ Set-up $\rightarrow$ & Bare probe (mSv/hr) & $\begin{array}{c}\text { Simulated patient (mSv/ } \\
\mathbf{h r})\end{array}$ & $\begin{array}{c}\text { Simulated patient \& shielding } \\
\text { (mSv/hr) }\end{array}$ \\
\hline Edge of shield & 17.4 & 4 & 0.121 \\
\hline 1metre from source & 14.3 & 0.38 & 0.065 \\
\hline Behind Anaesthetic room door & 0.074 & 0.07 & 0.003 \\
\hline Behind wall in prep room & 1.6 & 0.08 & 0.0004 \\
\hline Behind shield & $0.03-0.060$ & $0.011-0.048$ & 0.0052 \\
\hline Outside theatre door & 0.22 & 0.018 & 0.006 \\
\hline
\end{tabular}

Table 1: Results at some example points as a comparison of different set-ups.

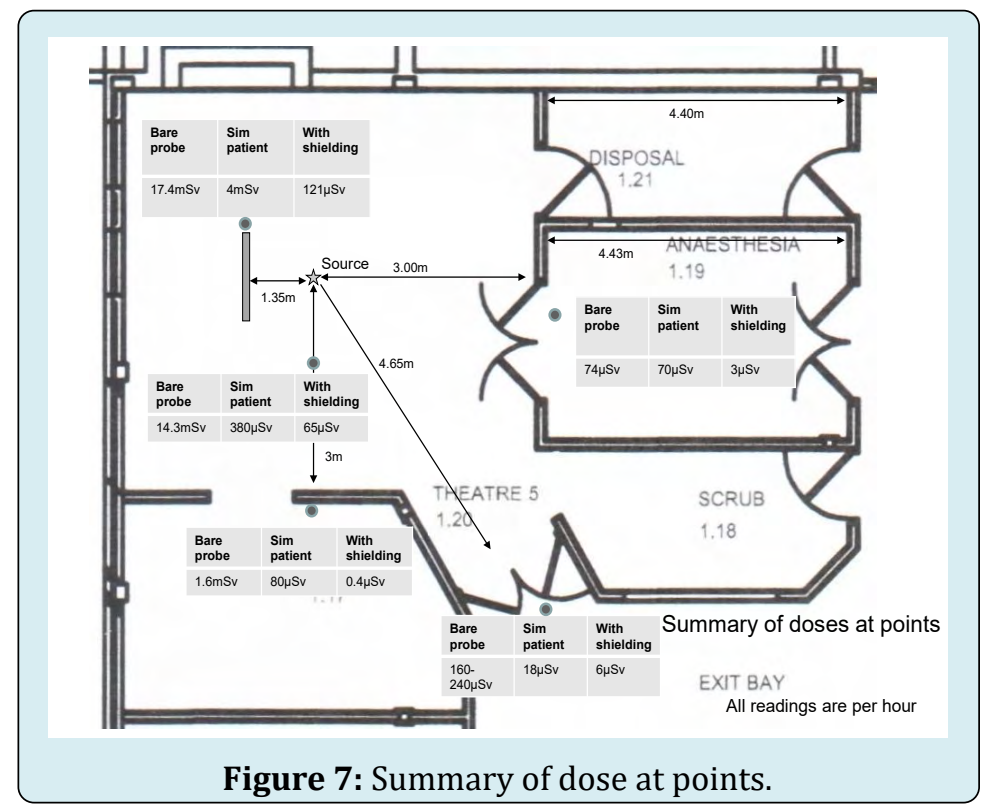




\section{Clinical Radiology \& Imaging Journal}

These positions are shown in the diagram below (without doses behind shield) to demonstrate the decision to increase shielding at each stage to reduce the dose to acceptable levels (Figure 7).

The surveys produced results in keeping with each decision made - a simulated patient provided attenuation, as did the use of lead equivalent shielding. With 2-4 expected patients a month, the TADR would have been acceptable, but the instantaneous dose rates were considered quite high still. Following these surveys and in accordance with the ALARP principle a decision was made to place additional shielding between the source and the anaesthetic and main theatre room doors. This reduced the dose rates to an acceptable level in the important locations (behind the primary shield, lobby area and anaesthetic room).

\section{Radiation Survey}

The image of the dose survey on the $24^{\text {th }}$ February 2014 shows the results of a repeat room survey when additional shielding was used. It shows that the dose rates within the room can be quite high (over $30 \mu \mathrm{Sv} / \mathrm{hr}$ ). Without the shielding, readings up to $7 \mu \mathrm{Sv} / \mathrm{hr}$ were recorded. Due to the location of the anaesthetic equipment staff had to stay on the far side of the room. It should be noted this room survey was under simulated treatment conditions - i.e. using breast equivalent material and shielding. As described a 'worst case scenario' (i.e. just the probe and applicator) gave rise to much higher readings $-214 \mu \mathrm{Sv} / \mathrm{hr}$ at the lobby and $30 \mu \mathrm{Sv} / \mathrm{hr}$ in the corridor outside the anaesthesia room. These readings show the advantages of using shielding around the source, and for additional shielding if required for certain public areas. Normally the shielding is placed between the entrance and the source, but certain factors (in this case location of anaesthetic gases) prevented this. Zeiss recommend the use of a $2.5 \mathrm{~mm}$ lead equivalent shield for the operators, and under normal treatment conditions the dose behind the shield was reduced almost to background. To optimise the layout, a room plan was produced showing the location of the equipment used, so that the additional shielding could be placed correctly. This meant certain equipment was placed differently to a normal theatre layout, but any issues were resolved during practice 'dry runs'. The location and alignment of the shields with regard to the source and the areas they are protecting is always checked prior to exposure (Figure 8).

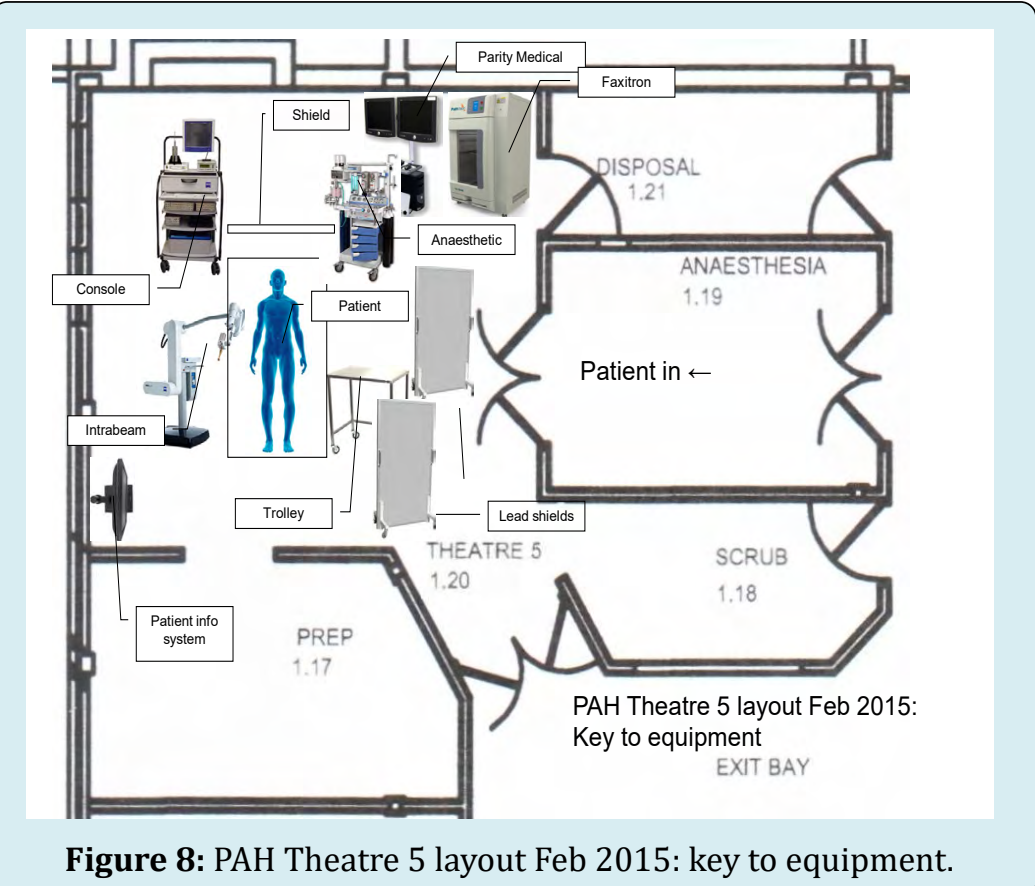

Commissioning of the system, which includes output measurements, isotropy around the probe and applicators and additional checks takes a few days. Generally results compare favourably enough with those provided by Zeiss that no changes are required.

\section{Linearity of Output with Time}

A 40 minute irradiation at $5 \mu \mathrm{A}$ using the bare probe in the water phantom was paused at 5 minute intervals $( \pm 1$ second). The dosemeter output (picocoulomb) and displayed dose (Gy) were recorded (Graph 1). 


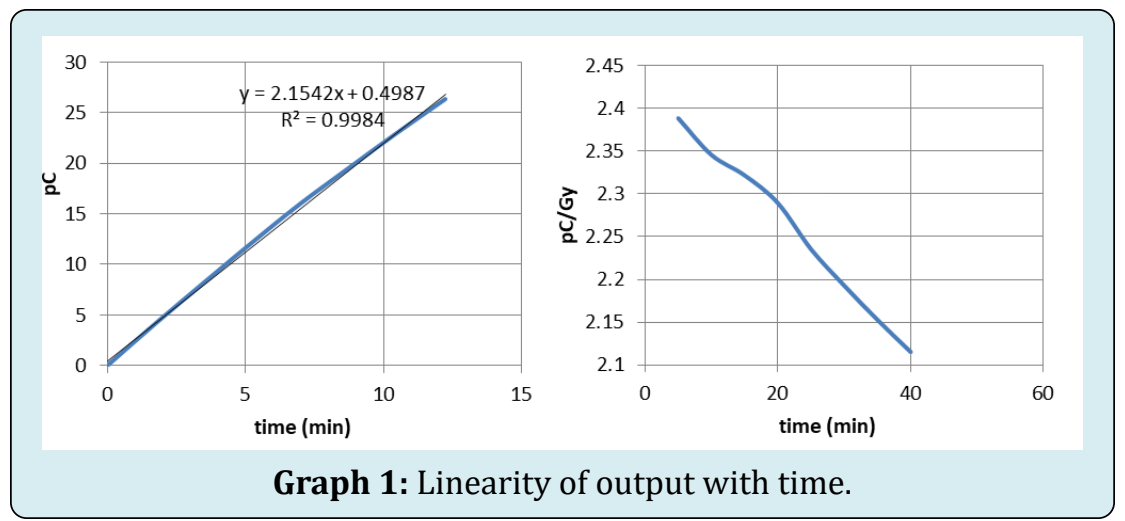

The apparent nonlinearity and decrease in dose meter sensitivity are consistent with heating of the water phantom and dose meter chamber throughout the exposure.

\section{Rotational Symmetry}

The bare probe and all applicators were placed in the Zeiss water phantom with the probe tip aligned with the centre of the laterally-placed ionisation chamber. Deviation of measured dose rate from the mean was plotted for successive 45 degree rotations of each chamber. The numbers refer to the serial numbers of the applicators. You would prefer a straight line showing no deviation, but these are acceptable results (Graph 2-4).
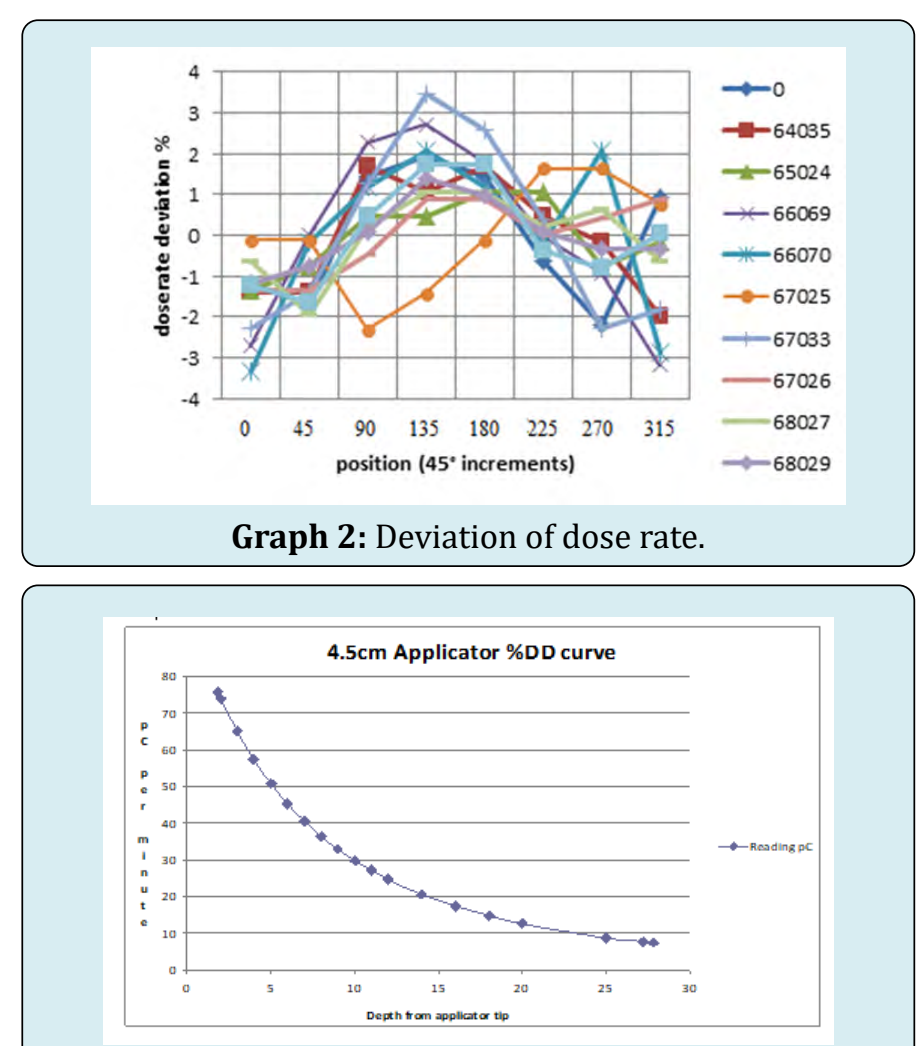

Graph 3: Depth dose data for a $4.5 \mathrm{~cm}$ applicator.

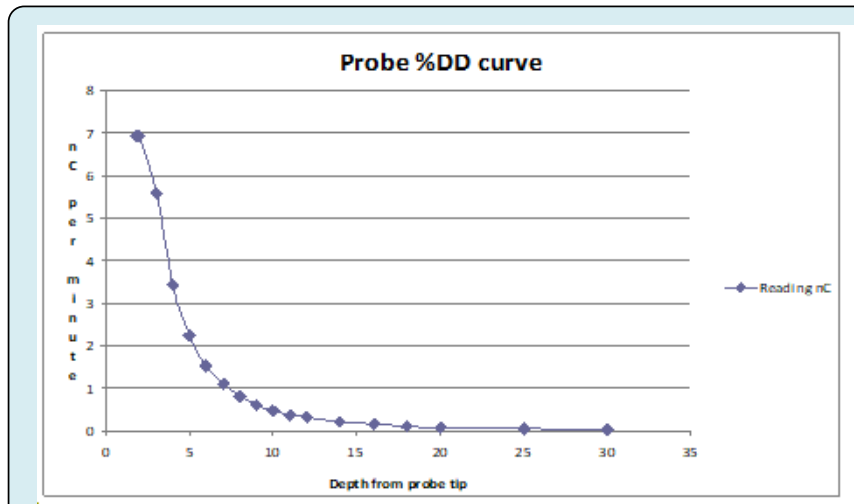

Graph 4: Depth dose curve for the bare probe.

This shows good agreement with manufactures specifications.

Data is obtained for both the bare probe and all of the applicators. This data is obtained using the water tank and a separate ionisation chamber. We can see that the drop in dose for the bare probe is much sharper than that with the $4.5 \mathrm{~cm}$ applicator and this varies with each applicator size why it is important that regular calibration of the depth dose is performed. Since the probe cannot touch the ionisation chamber while in an applicator there is a gap at the top of the curve, and the size of the water tank controls the achievable depth.

\section{Scatter Measurements; Effects of Breast Shield, Lead Screens}

Under IRR17 it is a legal requirement to monitor the staff who remain in the controlled area (physicist, radiotherapy radiographer and anaesthetist). Staff doses behind the shield tend to be negligible $(1-2 \mu \mathrm{Sv}$ per treatment), though scatter from surrounds should be considered and monitored to ensure the shield is placed correctly. If technetium is used for sentinel nodes then this may require additional monitoring depending on the activity used, care when handling the samples and disposal of the radioactive clinical waste. 


\section{Clinical Radiology \& Imaging Journal}

Generally the injected activity does not require controlled area designation, so local rules are not required - but the procedures could be incorporated into the local rules for Targit IORT.

Room layout is an issue, due to the additional equipment required as discussed earlier. A dry run is recommended not only to decide the best layout especially with the extra equipment required, but to familiarise staff with the procedure and changes to their normal theatre practices.

\section{Problems and Solutions; Practical Issues}

Controlling Access: Staff in theatres are used to entering rooms to get equipment, even while surgery is occurring. Lockable doors are recommended as signage may be ignored. Placing the Applicator over the Probe: For accurate treatment the probe must be aligned properly and if knocked will require straightening and additional QA which can lead to 20-30 minutes delay. A series of QA checks are carried out prior to treatment that checks the probe alignment to within $1 \mathrm{~mm}$, the isotropy of the beam and its output. Probe alignment is done using an inbuilt optical system, while output is checked by a chamber in the base of the equipment. If an issue arises, the QA must be redone. Therefore it is important that care is always taken with the probe and that the surgeons practice placing the applicator over the probe, in addition it is important to remember that while care is needed the procedure in performed in a short time as possible since longer the process the greater the deviation in alignment, calibration alignment shown below, the deviation can be up to $0.1 \mathrm{~mm}$ in the xy direction from a delay of $15 \mathrm{~min}$. Delay will lead to misalignment and can lead to the alignment being out of calibration and unacceptable by the system leading to the need for recalibration with the implication of delay to patient treatment, anaesthetic related issues and theatre time allocations (Figure 9).

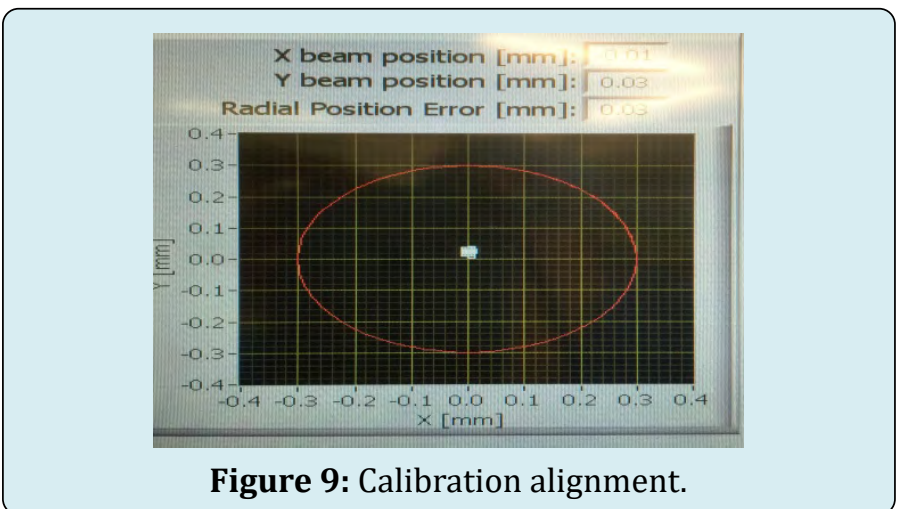

During treatment the beam alignment is constantly checked by an ion chamber monitoring the x-rays directed towards it. Alignment should be within $\pm .25 \mathrm{~mm}$ of the centre of the probe (shown as the centre of the cross hairs), while the series of white dot shows where the beam is actually hitting. This generates a series of dots that should be placed closely together and within the tolerance limit.

Choosing the Right Size Applicator: Until the surgeon places an applicator in the wound they will not know the exact size, and this can lead to several applicators being tried. As it is recommended each applicator is replaced after 100 sterilisations we recommend using steel or plastic sterilised balls of the same size to check which applicator would be required.

Communication: Since all staff have to be trained in radiation safety it is important that only trained staff are booked. The sterilisation team must be informed so that the applicators are ready in time - and can possibly complete sterilisation quickly for am and pm sessions if there are not enough of commonly used applicators. Depending on the set-up other staff groups must be kept informed, such as the radiotherapy radiographer and medical physics expert (MPE). IORT consumables should be added to the list of theatre consumables. It is also important to get key staff on board in the early stages. We advise developing a communication and decision tree so the correct staffs are informed at each stage.

Data Security: Patient data is stored on the console, but unless the console is kept secure patient data should be removed to a secure system as soon as possible (and remember deleted files are still present in the recycle bin).

\section{Discussion}

About $25 \%$ of cancers are breast cancer. Conventional external beam radiotherapy has to take place at radiotherapy centres, which may be some distance from the patient. Attending for CT scans, and 3 weeks of treatment can be a real effort both for the patient and their carer's. Though IORT is considered to be 'no better' or 'non-inferior' than external beam radiation therapy, from a hospital and patient point of view there can be considerable advantages. IORT requires some set up and commissioning work, but significantly less than for an external beam facility. A suitable theatre is required though, preferably at the edge of a building and certainly with controlled access. Shielding requirements tend to be in the tens of thousands of sterling and include 1 or 2 mobile lead screens as opposed to that required by a Linac. Staff tends to adapt to the new procedure very well, and the patients who have chosen the procedure seem more than happy. An obvious issue is trained staff - many nonradiotherapy centres do not have a therapy radiographer and a radiotherapy medical physics expert. However radiographers can be cross trained if required, especially 
Clinical Radiology \& Imaging Journal

if they are experienced in diagnostic work. The cost can be steep at first, but assessments show that the service would be profitable within 3-4 years depending on patient numbers, and this does not account for the savings made from not paying for external beam radiotherapy. It has been shown at several UK centres that there is no real impediment to implementing this service, with a minimum of difficulties.

\section{Conclusions}

It has been shown that IORT can be successfully implemented in small satellite centres, offering both hospitals and patients an alternative to external beam IORT. Communication can be considered vital to success, along with early staff involvement. Many staff are involved and it is important to have everyone on board, from sterilisation to administration staff who plan the rotas as well as other theatre staff. The IORT procedure can be appealing to private patients and therefore an income source, but concerns about this may require addressing. The additional theatre time required has to be balanced against income lost from normal breast surgery and off-set against savings in external beam radiotherapy as well as benefits to the patient. Setting up is similar to other procedures involving ionising radiation in that legislation must be considered and met, including critical exams, room surveys, risk assessments, commissioning suitable staff appointments and so on - but these are not too onerous. If staff are keen and experienced staff are involved then most issues can be quickly overcome.

The procedure has been shown to be suitable for small satellite centres, though suitable MPE cover can be an issue (which can be provided remotely). Theatre staff adapts quickly to the changes and perhaps most importantly of all the patients not only benefit but seems to prefer it.
Acknowledgements: Kind thanks to Princess Alexandra Hospital and Great Western hospitals for their assistance.

\section{References}

1. NICE (2018) Breast cancer (early)- intrabeam radiotherapy system: appraisal consultation document. National Institute for Health and Care Excellence.

2. RCR (2014) Multiple technology appraisal (MTA) INTERABEAM photon radiosurgery system for adjuvant treatment of early breast cancer. Clinical Oncology The Royal Collage of Radiologists.

3. Vaidya JS, Wenz F, Bulsara M, Tobias JS, Joseph DJ, et al. (2014) Risk-adapted targeted intraoperative radiotherapy versus whole-breast radiotherapy for breast cancer: 5-year results for local control and overall survival from the TARGIT-A randomised trial. Lancet 383(9917): 603-613.

4. Darby SC, Ewertz M, McGale P, Bennet AM, BlomGoldman U, et al. (2013) Risk of Ischemic Heart Disease in Women after Radiotherapy for Breast Cancer. N Engl J Med 368(11): 987-998.

5. Intraoperative radiotherapy systems, Carl Zeiss Meditec AG.

6. (2017) The Ionising radiation Regulations 2017 (IRR17), UK Statutory Instruments, legislation.gov.uk, No. 1075.

7. (2017) Ionising Radiations (Medical Exposure) Regulations 2017 (IR(ME)R2017), UK Statutory Instruments, legislation.gov.uk, No. 1322.

Advances in knowledge: first report on the practical aspects of setting up IORT for breast tumour treatment in a nonradiotherapy site.

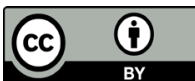

